\title{
BOUNDEDNESS OF AVERAGING OPERATORS ON GEOMETRICALLY DOUBLING METRIC SPACES
}

\author{
Jesús M. Aldaz \\ Universidad Autónoma de Madrid, Instituto de Ciencias Matemáticas (CSIC-UAM-UC3M-UCM) \\ and Departamento de Matemáticas, Cantoblanco 28049, Madrid, Spain; jesus.munarriz@uam.es
}

\begin{abstract}
We prove that averaging operators are uniformly bounded on $L^{p}$ for all geometrically doubling metric measure spaces and all $1 \leq p<\infty$, with bounds independent of the measure. From this result, the $L^{p}$ convergence of averages as $r \rightarrow 0$ immediately follows.
\end{abstract}

\section{Introduction}

It is a well known consequence of translation invariance that for Lebesgue measure on $\mathbf{R}^{d}$, the averages $A_{r} f$ (also known as Steklov means) converge to $f$ in $L^{1}$ as $r \rightarrow 0$. Rather surprisingly, the corresponding approximation question regarding arbitrary locally finite Borel measures $\mu$ on $\mathbf{R}^{d}$ (i.e., whether $\lim _{r \rightarrow 0} A_{r, \mu} f=f$ in $L^{1}(\mu)$ ) does not seem to have been studied in the literature.

Here we give an affirmative answer not just for $\mathbf{R}^{d}$, but for every geometrically doubling metric measure space (every metric space of homogeneous type, in the terminology of [CoWe], cf. Definition 2.5 below). We do this by proving that averaging operators are uniformly bounded on $L^{1}$, something that was previously unknown even for $\mathbf{R}^{d}$. By interpolation, boundedness also holds for all $p \in(1, \infty)$, since the case $p=\infty$ is trivial. Note that on $\mathbf{R}^{d}$ and for $p \in(1, \infty)$, the $L^{p}$ boundedness of averaging operators was already known, since it is immediate from the corresponding boundedness of the centered Hardy-Littlewood maximal operator. But for geometrically doubling metric spaces in which the Besicovitch covering theorem does not hold, not only the case $p=1$, but also the case $p \in(1, \infty)$, is new.

The boundedness question regarding euclidean spaces was asked by the author in [Al2]; two special cases were proved in that paper: the exponential distribution in one dimension, and the standard gaussian distribution in every dimension. Here we present the general result.

\section{Definitions and notation}

We will use $B^{o}(x, r):=\{y \in X: d(x, y)<r\}$ to denote open balls, and $B^{c l}(x, r):=$ $\{y \in X: d(x, y) \leq r\}$ to refer to metrically closed balls ("closed ball" will always be understood in the metric, not the topological sense). If we do not want to specify whether balls are open or closed, we write $B(x, r)$. But when we utilize $B(x, r)$, we assume that all balls are of the same kind, i.e., all open or all closed.

https://doi.org/10.5186/aasfm.2019.4430

2010 Mathematics Subject Classification: Primary 41A35.

Key words: Averaging operators, goemetrically doubling metric spaces.

The author was partially supported by Grant MTM2015-65792-P of the MINECO of Spain, and also by by ICMAT Severo Ochoa project SEV-2015-0554 (MINECO). 
Definition 2.1. A Borel measure is $\tau$-additive or $\tau$-smooth, if for every collection $\left\{U_{\alpha}: \alpha \in \Lambda\right\}$ of open sets,

$$
\mu\left(\bigcup_{\alpha} U_{\alpha}\right)=\sup _{\mathcal{F}} \mu\left(\bigcup_{i=1}^{n} U_{\alpha_{i}}\right),
$$

where the supremum is taken over all finite subcollections $\mathcal{F}=\left\{U_{\alpha_{1}}, \ldots, U_{\alpha_{n}}\right\}$ of $\left\{U_{\alpha}: \alpha \in \Lambda\right\}$. We say that $(X, d, \mu)$ is a metric measure space if $\mu$ is a $\tau$-additive Borel measure on the metric space $(X, d)$, such that $\mu$ assigns finite measure to bounded Borel sets.

From now on we always assume that measures are locally finite (finite on bounded sets) and not identically 0 . For motivation regarding the definition of metric measure spaces using $\tau$-additivity, cf. [Al3]. Note that in separable metric spaces all Borel measures are $\tau$-additive (so the spaces considered above are more general than those given by some commonly used alternative definitions, cf. [HKST] for instance) and the same happens with all Radon measures in arbitrary metric spaces. Recall that the complement of the support $(\operatorname{supp} \mu)^{c}:=\bigcup\left\{B^{o}(x, r): x \in X, \mu B^{o}(x, r)=0\right\}$ of a Borel measure $\mu$, is an open set, and hence measurable.

Definition 2.2. Let $(X, d)$ be a metric space and let $\mu$ be a locally finite Borel measure on $X$. If $\mu(X \backslash \operatorname{supp} \mu)=0$, we say that $\mu$ has full support.

By $\tau$-additivity, if $(X, d, \mu)$ is a metric measure space, then $\mu$ has full support, since $X \backslash \operatorname{supp} \mu$ is a union of open balls of measure zero. Actually, the other implication also holds, for the support is always separable, so having full support is equivalent to $\tau$-additivity (cf. [Bo, Proposition 7.2.10] for more details).

Definition 2.3. Let $(X, d, \mu)$ be a metric measure space and let $g$ be a locally integrable function on $X$. For each fixed $r>0$ and each $x \in \operatorname{supp} \mu$, the averaging operator $A_{r, \mu}$ is defined as

$$
A_{r, \mu} g(x):=\frac{1}{\mu(B(x, r))} \int_{B(x, r)} g d \mu .
$$

Averaging operators in metric measure spaces are defined almost everywhere, by $\tau$-additivity. Sometimes it is convenient to specify whether balls are open or closed; in that case, we use $A_{r, \mu}^{o}$ and $A_{r, \mu}^{c l}$ for the corresponding operators. Furthermore, when we are considering only one measure $\mu$ we often omit it, writing $A_{r}$ instead of the longer $A_{r, \mu}$.

Definition 2.4. Let $(X, d)$ be a metric space. A strict $r$-net (resp. non-strict $r$-net) in $X$ is a subset $S \subset X$ such that for any pair of distinct points $x, y \in S$, we have $d(x, y)>r($ resp. $d(x, y) \geq r)$.

We speak of an $r$-net if we do not want to specify whether it is strict or not. To ensure disjointness of the balls $B(x, r / 2), r$-nets are always taken to be strict when working with closed balls; otherwise, we assume $r$-nets are non-strict.

Definition 2.5. A metric space is geometrically doubling if there exists a positive integer $D$ such that every ball of radius $r$ can be covered with no more than $D$ balls of radius $r / 2$. We call the smallest such $D$ the doubling constant of the space.

We use $D^{o}$ and $D^{c l}$ to refer to the corresponding constants for open and for closed balls. It is easy to see, by enlarging balls slightly, that the geometrically doubling condition is satisfied for open balls if and only if it is satisfied for closed balls. But the constants will in general be different; for instance, if $X=\mathbf{R}$, then $D^{o}=3$ and $D^{c l}=2$. 
Remark 2.6. Let $X$ be geometrically doubling with constant $D$, and let $M$ be the maximum size of an $r$-net in $B(x, r)$, taken over all $x \in X$ and all $r>0$. Then $M \leq D$, since every point in a maximal $r$-net inside $B(x, r)$ is contained in one of the covering balls of radius $r / 2$, and each such ball can contain at most one point from the $r$-net. By analogy with previous notation, we use $M^{c l}$ and $M^{o}$ for strict and non-strict nets respectively.

\section{Boundedness of averaging operators on geometrically doubling spaces}

The following proof reminds the reader of the fact that bounded continuous functions with bounded support are dense in $L^{p}$ for $1 \leq p<\infty$, and for these functions, averages converge in norm as $r \rightarrow 0$.

Theorem 3.1. ( $L^{p}$-Lebesgue differentiation) Let $(X, d, \mu)$ be a metric measure space, and let $1 \leq p<\infty$. If there is a constant $C>0$ such that $\sup _{r>0}\left\|A_{r}\right\|_{L^{p}(\mu) \rightarrow L^{p}(\mu)}$ $\leq C$, then for every $f \in L^{p}(\mu), \lim _{r \rightarrow 0} A_{r} f=f$ in $L^{p}$.

Proof. Given any sequence $\left\{r_{n}\right\}_{n \geq 1}$ satisfying $r_{n} \rightarrow 0$, we may suppose that $r_{n} \leq 1$, by disregarding a finite number of terms, if needed. We may also suppose that $C \geq 1$ (else, replace it by 1 ).

Recall that in metric measure spaces the continuous functions that belong to $L^{p}$ are dense in $L^{p}$, by the standard argument whereby the case of real valued functions is reduced to the case of non-negative functions, which by successive approximations is reduced first, to the case of simple functions, then to indicator functions of measurable sets, and finally, to indicator functions of closed sets $F$ (with finite measure); for these functions the result is true by the Tietze-Urysohn extension theorem: given $\varepsilon>0$ we choose $O$ open such that $F \subset O$ and $\mu(O \backslash F)<\varepsilon$; then we extend $g: O^{c} \cup F \rightarrow\{0,1\}$ given by $g=1$ on $F, g=0$ on $O^{c}$, to a continuous function $G: X \rightarrow[0,1]$.

Let $z \in X$, let $\varepsilon>0$, and let $f \in L^{p}(\mu)$. Then there exist $t \gg 0$ and $R \gg 0$ such that

$$
\left\|f-f \mathbf{1}_{B(z, R) \cap\{|f| \leq t\}}\right\|_{p}<\frac{\varepsilon}{6 C} .
$$

Next we choose a continuous function $g$ such that $-t \leq g \leq t$, supp $g \subset B(z, R+1)$ and

By the triangle inequality,

$$
\left\|f \mathbf{1}_{B(z, R) \cap\{|f| \leq t\}}-g\right\|_{p}<\frac{\varepsilon}{6 C} .
$$

$$
\|f-g\|_{p}<\frac{\varepsilon}{3 C}
$$

Now $g$ is continuous, so for all $x \in X, \lim _{n} A_{r_{n}} g(x)=g(x)$. Since for all $n \geq 1$,

$$
\left|A_{r_{n}} g(x)-g(x)\right|^{p} \leq(2 t)^{p} \mathbf{1}_{B(z, R+2)}(x) \in L^{1}(\mu),
$$

by the dominated convergence theorem, $\lim _{n}\left\|A_{r_{n}} g-g\right\|_{p}=0$. Hence,

$$
\begin{aligned}
\limsup _{n}\left\|A_{r_{n}} f-f\right\|_{p} & \leq \sup _{n}\left\|A_{r_{n}} f-A_{r_{n}} g\right\|_{p}+\lim _{n}\left\|A_{r_{n}} g-g\right\|_{p}+\|g-f\|_{p} \\
& <\frac{\varepsilon}{3}+\frac{\varepsilon}{3 C}<\varepsilon .
\end{aligned}
$$

Definition 3.2. We call

$$
a_{s}(y):=\int_{X} \frac{\mathbf{1}_{B(y, s)}(x)}{\mu B(x, s)} d \mu(x)
$$

the conjugate function to the averaging operator $A_{s}$. 
As it happens with averaging operators, the conjugate function $a_{s}$ is well defined a.e., when $y$ belongs to the support of $\mu$. If one wishes, it can be defined everywhere via the usual conventions $0 / 0=0 \cdot \infty=0$, and $1 / 0=\infty$. These handle all the cases where the denominator vanishes.

Theorem 3.3. Let $(X, d, \mu)$ be a metric measure space. The averaging operator $A_{s}$ is bounded on $L^{1}(\mu)$ if and only if $a_{s} \in L^{\infty}(\mu)$, in which case $\left\|A_{s}\right\|_{L^{1}(\mu) \rightarrow L^{1}(\mu)}=$ $\left\|a_{s}\right\|_{\infty}$.

Proof. Let $0 \leq f \in L^{1}(\mu)$, and suppose $a_{s} \in L^{\infty}(\mu)$. Since by Fubini-Tonelli

$$
\begin{aligned}
\left\|A_{s} f\right\|_{L^{1}} & =\int_{X} A_{s} f(x) d \mu(x)=\int_{X} \int_{X} \frac{\mathbf{1}_{B(x, s)}(y)}{\mu B(x, s)} f(y) d \mu(y) d \mu(x) \\
& =\int_{X} f(y) \int_{X} \frac{\mathbf{1}_{B(y, s)}(x)}{\mu B(x, s)} d \mu(x) d \mu(y)=\int_{X} f(y) a_{s}(y) d \mu(y),
\end{aligned}
$$

it follows from Hölder's inequality that $\left\|A_{s}\right\|_{L^{1}(\mu) \rightarrow L^{1}(\mu)} \leq\left\|a_{s}\right\|_{\infty}$.

On the other hand, we claim that if $\left\|A_{s}\right\|_{L^{1}(\mu) \rightarrow L^{1}(\mu)} \leq C$, then $\left\|a_{s}\right\|_{\infty} \leq C$. Towards a contradiction, suppose $C<\left\|a_{s}\right\|_{\infty}$ (including the case $\left\|a_{s}\right\|_{\infty}=\infty$ ). Then there is a $t>C$ and a measurable set $A_{t}$ such that $A_{t} \subset\left\{a_{s}>t\right\}$ and $0<\mu A_{t}<\infty$. Let $f:=\mathbf{1}_{A_{t}} \in L^{1}(\mu)$. Then

$$
\left\|A_{s} f\right\|_{L^{1}}=\int_{X} f(y) a_{s}(y) d \mu(y)>\int_{A_{t}} t d \mu(y)=t \mu\left(A_{t}\right)>C\|f\|_{L^{1}} .
$$

Definition 3.4. We say that a measure $\mu$ satisfies a local comparability condition if there exists a constant $C \in[1, \infty)$ such that for all pairs of points $x, y \in X$ and every $r>0$, whenever $d(x, y)<r$, we have $\mu(B(x, r)) \leq C \mu(B(y, r))$.

The preceding definition comes from [NaTa, p. 737]. There, local comparability is called a "mild uniformity assumption"; the term "local comparability" was introduced in [Al2], and used also in [Al1]. As indicated in [NaTa, p. 737], if $\mu$ satisfies a $C$ local comparability condition, then $a_{s}(y) \leq C$, so $\left\|A_{s}\right\|_{L^{1} \rightarrow L^{1}} \leq C$.

It is natural to ask under which conditions one can have uniform boundedness of $A_{r}$ without local comparability. In [Al2, Example 4.1] a metric measure space is exhibited where the measure lacks local comparability, and $\left\|A_{s}\right\|_{L^{p} \rightarrow L^{p}}$ is unbounded for all $p \in[1, \infty)$. Also, it is shown in $[\mathrm{Al} 2$, Theorem 4.8] that on $\mathbf{R}$, there is a measure $\mu$ such that the right directional averaging operator

$$
A_{s, \mu}^{r} f(x):=\frac{1}{\mu([x, x+s])} \int_{[x, x+s]} f(y) d \mu(y)
$$

is unbounded on $L^{1}(\mu)$ for $s=1$.

On the other hand, the uniform $L^{1}$ boundedness of the operators $A_{r}$ was shown to hold for $\mathbf{R}^{d}$ in two special cases, despite the lack of local comparability: the exponential density in dimension one, and the standard gaussian measures in every dimension (cf. [Al2, Theorems 4.2 and 4.3]).

Here we obtain the general result: Averaging operators are $L^{1}$ bounded, uniformly on $r$, for every locally finite measure in any geometrically doubling metric measure space, not just in $\mathbf{R}^{d}$.

Theorem 3.5. Let $(X, d, \mu)$ be a geometrically doubling metric measure space, with doubling constant $D$, and let $M$ be the maximum cardinality of any $r$-net in $B(x, r)$, where the maximum is taken over all $x \in X$ and all $r>0$. Then for every 
$s>0,\left\|A_{s}\right\|_{L^{1}(\mu) \rightarrow L^{1}(\mu)} \leq M \leq D$. Since $\left\|A_{s}\right\|_{L^{\infty}(\mu) \rightarrow L^{\infty}(\mu)}=1$, for $1<p<\infty$ we have $\left\|A_{s}\right\|_{L^{p}(\mu) \rightarrow L^{p}(\mu)} \leq M^{1 / p}$ by interpolation.

Proof. Let $X$ be geometrically doubling with constant $D$, and let $M$ be the maximum size of an $r$-net in $B(x, r)$. As was noted before, $M \leq D$. By disregarding a set of measure zero if needed, we suppose that $X=\operatorname{supp} \mu$, so every ball has positive measure. Fix $y \in X$. We want to show that $a_{s}(y) \leq M$, and then the result follows from Theorem 3.3.

First we claim that $b_{1}:=\inf \{\mu B(x, s): x \in B(y, s)\}>0$. To see why, select a sequence $\left\{x_{n}\right\}_{n \geq 1}$ of points in $B(y, s)$ so that $\lim _{n} \mu B\left(x_{n}, s\right)=b_{1}$. Since $X$ is geometrically doubling, $B(y, s)$ can be covered by at most $D$ balls of radius $s / 2$, so at least one of these balls, say, $B(w, s / 2)$, contains an infinite subsequence from $\left\{x_{n}\right\}_{n \geq 1}$, which after relabelling, we also denote by $\left\{x_{n}\right\}_{n \geq 1}$. Then $b_{1} \geq \mu B(w, s / 2)>0$, since for all $n \geq 1, B(w, s / 2) \subset B\left(x_{n}, s\right)$. Now take $0<\varepsilon \ll 1$, and choose $u_{1} \in B(y, s)$ so that $\mu B\left(u_{1}, s\right)<(1+\varepsilon) b_{1}$; let $b_{2}:=\inf \{\mu B(x, s): x \in B(y, s) \backslash$ $\left.B\left(u_{1}, s\right)\right\}$, and select $u_{2} \in B(y, s) \backslash B\left(u_{1}, s\right)$ so that $\mu B\left(u_{2}, s\right)<(1+\varepsilon) b_{2}$; repeat, with $b_{k+1}:=\inf \left\{\mu B(x, s): x \in B(y, s) \backslash \bigcup_{1}^{k} B\left(u_{i}, s\right)\right\}, u_{k+1} \in B(y, s) \backslash \bigcup_{1}^{k} B\left(u_{i}, s\right)$, and $\mu B\left(u_{k+1}, s\right)<(1+\varepsilon) b_{k+1}$. Since the points $u_{i}$ form an $s$-net in $B(y, s)$, there is an $m \leq M$ such that $B(y, s) \backslash \bigcup_{1}^{m} B\left(u_{i}, s\right)=\emptyset$, and then the process stops.

Next, fix $x \in B(y, s)$, and let $i$ be the first index such that $x \in B\left(u_{i}, s\right)$. Then

$$
\frac{\mathbf{1}_{B(y, s)}(x)}{\mu B(x, s)} \leq(1+\varepsilon) \frac{\mathbf{1}_{B(y, s) \cap B\left(u_{i}, s\right)}(x)}{\mu B\left(u_{i}, s\right)} \leq(1+\varepsilon) \sum_{j=1}^{m} \frac{\mathbf{1}_{B(y, s) \cap B\left(u_{j}, s\right)}(x)}{\mu B\left(u_{j}, s\right)},
$$

SO

$$
\begin{aligned}
a_{s}(y) & =\int_{X} \frac{\mathbf{1}_{B(y, s)}(x)}{\mu B(x, s)} d \mu(x) \leq \int_{X}(1+\varepsilon) \sum_{j=1}^{m} \frac{\mathbf{1}_{B(y, s) \cap B\left(u_{j}, s\right)}(x)}{\mu B\left(u_{j}, s\right)} d \mu(x) \\
& \leq(1+\varepsilon) \int_{X} \sum_{j=1}^{m} \frac{\mathbf{1}_{B\left(u_{j}, s\right)}(x)}{\mu B\left(u_{j}, s\right)} d \mu(x) \leq(1+\varepsilon) M,
\end{aligned}
$$

and $\left\|A_{s}\right\|_{L^{1}(\mu) \rightarrow L^{1}(\mu)} \leq M$ follows by letting $\varepsilon \downarrow 0$. It is obvious that for all $s>0$, $\left\|A_{s}\right\|_{L^{\infty}(\mu) \rightarrow L^{\infty}(\mu)}=1$ (we always have $\left\|A_{s}\right\|_{L^{\infty}(\mu) \rightarrow L^{\infty}(\mu)} \leq 1$, since averages never exceed a supremum; for the other inequality, just take $f=\mathbf{1}_{X}$ ). Thus, by a standard interpolation argument, or simply by Jensen's inequality (cf. [Al2, Theorem 2.10]) for all $1<p<\infty$ we have $\left\|A_{s}\right\|_{L^{p}(\mu) \rightarrow L^{p}(\mu)} \leq M^{1 / p}$.

While the bounds given in the preceding theorem do not seem very tight, they are. Adapting some arguments from [Al4], we show next that it is possible to have $\left\|A_{1, \mu}^{c l}\right\|_{L^{1}(\mu) \rightarrow L^{1}(\mu)}=M^{c l}=D^{c l}$, so the bounds from Theorem 3.5 cannot in general be improved. The exponential dependency on the dimension of the space was already known in some natural cases: for the standard gaussian measure $\gamma^{d}$ on $\left(\mathbf{R}^{d},\|\cdot\|_{2}\right)$, for $1 \leq p<\infty$, and for every $d$ sufficiently large, the weak type $(p, p)$ constants satisfy $\left\|A_{\frac{\sqrt{3 d-3}}{2}}\right\|_{L^{p} \rightarrow L^{p, \infty}}>1.019^{d / p}$, cf. [Al2, Theorem 4.3].

Recall that balls with respect to $\|\cdot\|_{\infty}$, the $\ell_{\infty}$ norm on $\mathbf{R}^{d}$, are cubes with sides parallel to the axes.

Theorem 3.6. There exists a discrete measure $\mu$ on $\left(\mathbf{R}^{d},\|\cdot\|_{\infty}\right)$ such that $\left\|A_{1, \mu}^{c l}\right\|_{L^{1}(\mu) \rightarrow L^{1}(\mu)}=2^{d}=M^{c l}=D^{c l}$.

Proof. That $2^{d}=M^{c l}=D^{c l}$ is clear: consider $B^{c l}(0,1)=[-1,1]^{d}$; its vertices form a strict 1 -net in $B^{c l}(0,1)$, so $2^{d} \leq M^{c l}$. Also, the translates of $[0,1]^{d}$ that are 
contained in $[-1,1]^{d}$ and share a vertex with $[-1,1]^{d}$ form a cover of $[-1,1]^{d}$, so $2^{d} \geq D^{c l}$.

Let $\left\{x_{1}, \ldots, x_{2^{d}}\right\}$ be an enumeration of the vertices of $[-3 / 4,3 / 4]^{d}$. For $n \geq 1$, set $\mu_{n}:=n^{-1} \delta_{3 n e_{1}}+\sum_{i=1}^{2^{d}} \delta_{x_{i}+3 n e_{1}}$, and let $\mu:=\sum_{n=1}^{\infty} \mu_{n}$. If $f_{n}:=n \mathbf{1}_{3 n e_{1}}$, then $\left\|f_{n}\right\|_{L^{1}(\mu)}=1$ and $\left\|A_{1, \mu}^{c l} f_{n}\right\|_{L^{1}(\mu)}>2^{d} n /(n+1)$.

Putting together Theorems 3.1, 3.3, and 3.5, we obtain the following

Corollary 3.7. Suppose $(X, d, \mu)$ is either a geometrically doubling metric measure space, or $\mu$ satisfies a local comparability condition. Then for every $f \in L^{p}(\mu)$, $1 \leq p<\infty$, we have $\lim _{r \rightarrow 0} A_{r} f=f$ in $L^{p}$.

Corollary 3.8. Suppose $(X, d, \mu)$ is either a geometrically doubling metric measure space, or $\mu$ satisfies a local comparability condition. Then for all $f \in L_{l o c}^{1}(\mu)$, $M f(x) \geq|f|(x)$ almost everywhere.

Proof. This follows from Corollary 3.7, since from $L^{1}$ convergent sequences one can always extract subsequences converging a.e., and for every $r>0, M f(x) \geq$ $A_{r}|f|(x)$.

The part of the preceding corollary dealing with geometrically doubling metric spaces had been originally obtained (cf. [Al3, Corollary 2.10]) by using a result of Hytönen (cf. [Hy, Lemma 3.3]) on the existence of arbitrarily small doubling balls, for general measures in geometrically doubling metric measure spaces.

In view of the exponential increase of the bounds, for the standard gaussian measure $\gamma^{d}$ on $\left(\mathbf{R}^{d},\|\cdot\|_{2}\right)$, one might suspect that in the infinite dimensional case the uniform boundedness of the averaging operators can fail. It follows from a result of Preiss that this is indeed the case.

Corollary 3.9. There is a gaussian measure $\gamma$ on an infinite dimensional separable Hilbert space $H$, for which $\sup _{r>0}\left\|A_{r}\right\|_{L^{p}(\gamma) \rightarrow L^{p}(\gamma)}=\infty$ whenever $1 \leq p<\infty$.

Proof. By $[\mathrm{Pr}]$, there exists a gaussian probability $\gamma$ on a separable Hilbert space $H$ and a Borel subset $C$ with $0<\gamma C<1$, such that $\gamma$-a.e. x,

$$
\lim _{r \rightarrow 0} \frac{\gamma(C \cap B(x, r))}{\gamma(B(x, r))}=0 .
$$

Fix $p \in[1, \infty)$. By Theorem 3.1, if $\sup _{r>0}\left\|A_{r}\right\|_{L^{p}(\mu) \rightarrow L^{p}(\mu)}<\infty$, then $\lim _{r \rightarrow 0} \| A_{r} \mathbf{1}_{C}-$ $\mathbf{1}_{C} \|_{p}=0$, so it is possible to extract a sequence $r_{n} \rightarrow 0$ such that $\gamma$ a.e. $x$,

$$
\lim _{n} \frac{\gamma\left(C \cap B\left(x, r_{n}\right)\right)}{\gamma\left(B\left(x, r_{n}\right)\right)}=\mathbf{1}_{C}(x)
$$

which is a contradiction.

\section{References}

[Al1] Aldaz, J. M.: The Stein-Strömberg covering theorem in metric spaces. - J. Math. Anal. Appl. 449:2, 2017, 1741-1753.

[Al2] ALDAZ, J. M.: Local comparability of measures, averaging and maximal averaging operators. - Potential Anal. 49:2, 2018, 309-330.

[Al3] AldAz, J. M.: On the pointwise domination of a function by its maximal function. - Arch. Math. (Basel) 111:3, 2018, 299-311.

[Al4] Aldaz, J. M.: Kissing numbers and the centered maximal operator. - Preprint, ArXiv.

[Bo] Bogachev, V. I.: Measure theory. - Springer-Verlag, 2017. 
[CoWe] Coifman, R. R., and G. Weiss: Analyse harmonique non-commutative sur certains espaces homogènes. Étude de certaines intégrales singulières. - Lecture Notes in Math. 242, Springer-Verlag, Berlin-New York, 1971.

[HKST] Heinonen, J., P. Koskela, N. Shanmugalingam, and J. T. Tyson: Sobolev spaces on metric measure spaces. An approach based on upper gradients. - New Math. Monogr. 27, Cambridge Univ. Press, Cambridge, 2015.

[Hy] Hytönen, T.: A framework for non-homogeneous analysis on metric spaces, and the RBMO space of Tolsa. - Publ. Mat. 54:2, 2010, 485-504.

[NaTa] NAOR, A., and T. TAO: Random martingales and localization of maximal inequalities. J. Funct. Anal. 259:3, 2010, 731-779.

[Pr] Preiss, D.: Gaussian measures and the density theorem. - Comment. Math. Univ. Carolin. 22:1, 1981, 181-193.

Received 5 June 2018 • Accepted 29 November 2018 